\title{
Nonlinear Scattering by Anisotropic Dielectric Periodic Structures
}

\author{
O. V. Shramkova and A. G. Schuchinsky \\ School of Electronics, Electrical Engineering and Computer Science, Queen's University Belfast, Queen's Road, Queen's Island, \\ Belfast BT3 9DT, UK \\ Correspondence should be addressed to O. V. Shramkova, o.shramkova@qub.ac.uk
}

Received 28 June 2012; Accepted 24 July 2012

Academic Editor: Ivan D. Rukhlenko

Copyright ( 2012 O. V. Shramkova and A. G. Schuchinsky. This is an open access article distributed under the Creative Commons Attribution License, which permits unrestricted use, distribution, and reproduction in any medium, provided the original work is properly cited.

The combinatorial frequency generation by the periodic stacks of binary layers of anisotropic nonlinear dielectrics is examined. The products of nonlinear scattering are characterised in terms of the three-wave mixing processes. It is shown that the intensity of the scattered waves of combinatorial frequencies is strongly influenced by the constitutive and geometrical parameters of the anisotropic layers, and the frequency ratio and angles of incidence of pump waves. The enhanced efficiency of the frequency conversion at Wolf-Bragg resonances has been demonstrated for the lossless and lossy-layered structures.

\section{Introduction}

A new generation of artificial electromagnetic materials has opened up new opportunities for engineering the media with the specified properties. The latest advancements in this field have prompted a surge of research in the new phenomenology, which could extend a range of functional capabilities and enable the development of innovative devices in the millimeter, terahertz (THz), and optical ranges.

Frequency conversion in dielectrics with nonlinearities of the second and third order has been investigated in optics, particularly, in the context of the second (SHG) and third (THG) harmonic generation. The recent studies have indicated that nonlinear photonic crystals (PhCs) and metamaterials (MMs) have significant potential for enhancement of the nonlinear activity associated with the mechanisms of field confinement, dispersion management and resonant intensification of the interacting waves. For example, it has been demonstrated in [1-5] that the PhCs dispersion can be tailored to facilitate the phase synchronism (The phase synchronism between pump wave and its harmonic is a prerequisite for efficient frequency conversion.) between the second harmonic and the pump wave of fundamental frequency. The harmonic generation efficiency can be further increased when the pump wave frequencies are close to the $\mathrm{PhC}$ band edges [6-12] where the higher density of states provides favourable phase-matching conditions. The SHG efficiency also grows with the PhC thickness or the number of stacked layers [5].

Combinatorial frequency generation by mixing pump waves of two different frequencies provides alternative means for frequency conversion. The efficiency of mixing process can be dramatically increased in the layered structures, for example, at the higher order Wolf-Bragg resonances of the combinatorial frequencies generated in the anisotropic nonlinear dielectric slabs. As shown in [13], at the specific thickness of the layers illuminated by the plane waves of two tones, the mixing products reach their extremes and exhibit either giant growth of the peak intensity or full suppression. The global maxima and nulls at Wolf-Bragg resonances in the layer are achieved only at the particular combinations of the two frequencies $\omega_{1,2}$ of pump waves and the layer parameters and anisotropy.

The aim of this paper is to explore the mechanisms of the combinatorial frequency generation in the $\mathrm{PhC}$ composed of a periodic stack of binary anisotropic nonlinear dielectric 


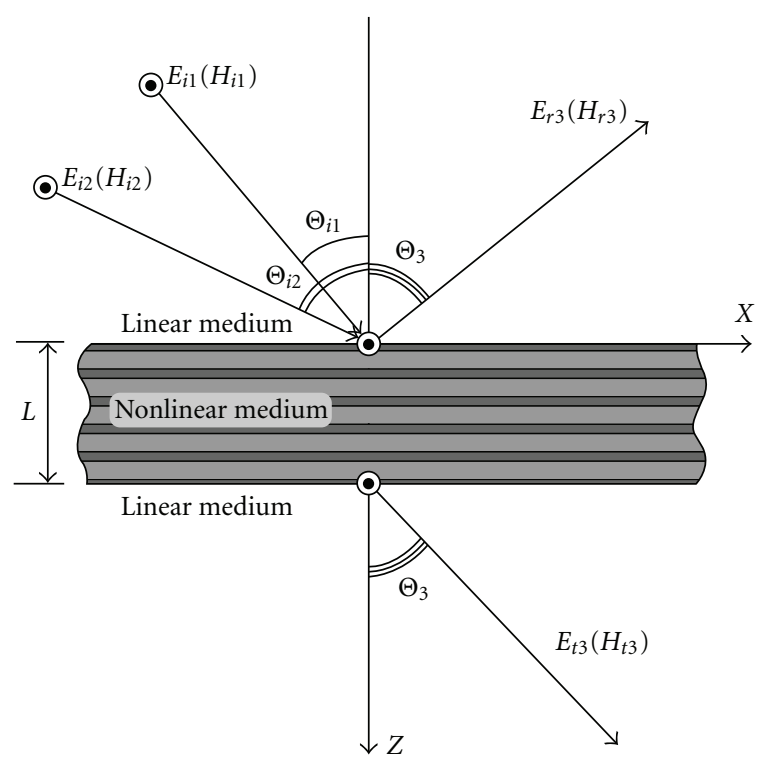

Figure 1: Geometry of the problem.

layers illuminated by two-tone pump waves that allows us to combine the effects of the resonance mixing with the dispersion control provided by the structure periodicity. Here the properties of the combinatorial frequencies generated by the nonlinear anisotropic dielectric $\mathrm{PhC}$ illuminated by plane waves of two tones are investigated. A generic approach, based on the transfer matrix method (TMM) [14], has been devised here to take into account nonlinear polarization of the constituent anisotropic layers and analyse frequency mixing of the two-tone plane waves obliquely incident on the PhC. The problem statement and the solution of the respective boundary value problem obtained in the three-wave approximation [15] are outlined in Section 2. The results of the numerical analysis and the properties of TM waves of combinatorial frequencies scattered by the nonlinear PhC are discussed in Section 3 and the main features of the three-wave mixing products generated by the anisotropic nonlinear PhCs are summarised in Conclusions.

\section{Nonlinear Scattering in Three-Wave Mixing Process}

Wave propagation and scattering in linear stratified media are usually modelled by TMM, which sequentially relates the fields at the layer interfaces, see, for example, $[14,16]$. The TMM approach has also been applied to the study of optical harmonic generation and frequency mixing in 1D nonlinearlayered structures at normal incidence of the pump waves [17-20]. The nondepleted pump wave approximation has been usually employed taking into account multiple reflections from the layer interfaces and interference between all propagating waves, including the forward and backward propagating waves. A relatively simple approach based upon the TMM generalisation to a multiwavelength case has been proposed in [19] where interaction between the different frequencies was described by the "effective" refractive index characteristic for each optical wave. The latter technique allows simulations of multiple optical wave interactions in the homogenised metamaterials as well as in PhCs.

In order to examine the three-wave mixing process in the $1 \mathrm{D}$ anisotropic $\mathrm{PhC}$, it is necessary to generalise the TMMbased analysis for the case of two pump waves, incident at arbitrary angles. To elucidate the main features of the developed approach, we consider here a canonical PhC structure with the cross-section shown in Figure 1. It is composed of the periodic binary dielectric layers of thicknesses $d_{1}$ and $d_{2}$ and infinite extent in the $x$ and $y$ directions. The total thickness of the periodic stack is $L=N \cdot\left(d_{1}+d_{2}\right)$, where $N$ is the number of periods (unit cells). The $\mathrm{PhC}$ is surrounded by the linear homogeneous medium with the dielectric permittivity $\varepsilon_{a}$ at $z \leq 0$ and $z \geq L$. It is illuminated by two plane waves of frequencies $\omega_{1}$ and $\omega_{2}$ incident at angles $\Theta_{i 1}$ and $\Theta_{i 2}$, respectively, as shown in Figure 1.

Each layer has $6 \mathrm{~mm}$ class of anisotropy and is described by the linear dielectric permittivity tensor $\hat{\varepsilon}=\left(\varepsilon_{x x}, \varepsilon_{x x}, \varepsilon_{z z}\right)$ and the second-order nonlinear susceptibility tensor $\hat{\chi}$ :

$$
\hat{\chi}=\left(\begin{array}{cccccc}
0 & 0 & 0 & 0 & \chi_{x x z} & 0 \\
0 & 0 & 0 & \chi_{x x z} & 0 & 0 \\
\chi_{z x x} & \chi_{z x x} & \chi_{z z z} & 0 & 0 & 0
\end{array}\right)
$$

Owing to the structure uniformity in the $x 0 y$ plane and symmetry of the tensors $\hat{\varepsilon}$ and $\hat{\chi}$, we can assume without loss of generality that $\partial / \partial y=0$. In this case, Maxwell's equations for TE and TM polarised waves are separated and can be treated independently. Only TM waves are considered in the rest of the paper (the analysis of TE waves is similar and somewhat simpler being unaffected by anisotropy of $\hat{\chi}$ defined in (1)). The electric $E_{x, z}$ and magnetic $H_{y}$ field components of TM waves in each layer satisfy the following system of nonlinear equations:

$$
\begin{gathered}
\frac{\partial E_{x j}}{\partial z}-\frac{\partial E_{z j}}{\partial x}+\frac{1}{c} \frac{\partial H_{y j}}{\partial t}=0 \\
\frac{\partial H_{y j}}{\partial z}+\frac{\varepsilon_{x x j}}{c} \frac{\partial E_{x j}}{\partial t}=-\frac{4 \pi}{c} \chi_{x x z j} \frac{\partial}{\partial t}\left(E_{x j} E_{z j}\right), \\
\frac{\partial H_{y j}}{\partial x}-\frac{\varepsilon_{z z j}}{c} \frac{\partial E_{z j}}{\partial t}=\frac{4 \pi}{c}\left[\chi_{z x x j} \frac{\partial}{\partial t}\left(E_{x j} E_{x j}\right)+\chi_{z z z j} \frac{\partial}{\partial t}\left(E_{z j} E_{z j}\right)\right],
\end{gathered}
$$

where $j=1,2$ denotes the respective constituent nonlinear layer in the binary unit cell, $c$ is the speed of light.

In the approximation of weak nonlinearity, the scattering characteristics of the TM waves can be obtained separately at each frequency by the harmonic balance method. Thus at the combinatorial frequency $\omega_{3}=\omega_{1}+\omega_{2}$, the system of nonlinear equations (2) can be reduced to inhomogeneous 
Helmholtz equation for $H_{y j}$ in each nonlinear anisotropic dielectric layer

$$
\begin{gathered}
\frac{\partial^{2} H_{y j}\left(\omega_{3}\right)}{\varepsilon_{x x j} \partial z^{2}}+\left(k_{3}^{2}-\frac{k_{x 3}^{2}}{\varepsilon_{z z j}}\right) H_{y j}\left(\omega_{3}\right) \\
=4 \pi k_{3}\left[2 \frac{\partial}{\partial x}\left(\frac{\chi z x x j}{\varepsilon_{z z j}} E_{x j}\left(\omega_{1}\right) E_{x j}\left(\omega_{2}\right)+\frac{\chi_{z z z j}}{\varepsilon_{z z j}} E_{z j}\left(\omega_{1}\right) E_{z j}\left(\omega_{2}\right)\right)\right. \\
\left.-\frac{\chi x x z j}{\varepsilon_{x x j}} \frac{\partial}{\partial z}\left(E_{x j}\left(\omega_{1}\right) E_{z j}\left(\omega_{2}\right)+E_{x j}\left(\omega_{2}\right) E_{z j}\left(\omega_{1}\right)\right)\right],
\end{gathered}
$$

where $k_{p}=\omega_{p} / c, p=1,2,3$ and $k_{x 3}=k_{3} \sqrt{\varepsilon_{a}} \sin \Theta_{3}$. Since $k_{x 3}$ must obey the requirement of the waveform invariance along the layer interfaces, the phase synchronism condition in the three-wave mixing process [15] is enforced here in the following form:

$$
k_{x 3}=k_{x 1}+k_{x 2}
$$

where $k_{x 1, x 2}=k_{1,2} \sqrt{\varepsilon_{a}} \sin \Theta_{i 1, i 2}$. In order to make the solution procedure more transparent, we assume here that both incident pump waves of frequencies $\omega_{1,2}$ have the same amplitudes equal to unity. Generalisation to the case of unequal pump wave amplitudes is straightforward but the resulting expressions are more cumbersome.

The full solution of inhomogeneous equation (3) is composed of the partial and general solutions which can be represented in the form

$$
\begin{aligned}
H_{y j}^{(n)}\left(\omega_{3}, x, z\right)= & \left(A_{j}^{n+} e^{i k_{z L j}^{(3)} z}+A_{j}^{n-} e^{-i k_{z L j}^{(3)} z}+D_{1 j}^{n+} e^{i k_{z L j}^{+} z}\right. \\
& \left.+D_{2 j}^{n+} e^{-i k_{z L j}^{+} z}+D_{1 j}^{n-} e^{i k_{z L j}^{-} z}+D_{2 j}^{n-} e^{-i k_{z L j}^{-} z}\right) \\
& \times e^{-i \omega_{3} t+i k_{x 3} x} .
\end{aligned}
$$

Here the amplitude coefficients $A_{j}^{n \pm}$ are associated with the general solution of (3) and are determined by means of enforcing the continuity conditions for the tangential field components at the layer interfaces. The coefficients $D_{1 j, 2 j}^{n \pm}$ represent the partial solution of inhomogeneous equation (3) and are expressed in terms of the refracted field amplitudes in each layer at the pump wave frequencies $\omega_{1}$ and $\omega_{2}$ :

$$
\begin{aligned}
& D_{1 j}^{n+}=\alpha_{j} \beta_{j} \frac{B_{j}^{n+}\left(\omega_{1}\right) B_{j}^{n+}\left(\omega_{2}\right)}{\left(k_{z L j}^{+}\right)^{2}-\left(k_{z L j}^{(3)}\right)^{2}}, \\
& D_{2 j}^{n+}=\alpha_{j} \beta_{j} \frac{B_{j}^{n-}\left(\omega_{1}\right) B_{j}^{n-}\left(\omega_{2}\right)}{\left(k_{z L j}^{+}\right)^{2}-\left(k_{z L j}^{(3)}\right)^{2}}, \\
& D_{1 j}^{n-}=\alpha_{j} \gamma_{j} \frac{B_{j}^{n+}\left(\omega_{1}\right) B_{j}^{n-}\left(\omega_{2}\right)}{\left(k_{z L j}^{-}\right)^{2}-\left(k_{z L j}^{(3)}\right)^{2}} \text {, } \\
& D_{2 j}^{n-}=\alpha_{j} \gamma_{j} \frac{B_{j}^{n-}\left(\omega_{1}\right) B_{j}^{n+}\left(\omega_{2}\right)}{\left(k_{z L j}^{-}\right)^{2}-\left(k_{z L j}^{(3)}\right)^{2}} \text {, } \\
& \alpha_{j}=\frac{4 \pi}{\varepsilon_{z z j}} \frac{k_{3}}{k_{1} k_{2}}, \\
& \beta_{j}=-k_{z L j}^{+} k_{x 1} k_{z L j}^{(2)} \frac{\chi_{x x z j}}{\varepsilon_{x x j}} \\
& -k_{x 3}\left(\frac{\chi_{z x x j}}{\varepsilon_{x x j}} k_{z L j}^{(1)} k_{z L j}^{(2)}+\frac{\chi_{z z z j} \varepsilon_{x x j}}{\varepsilon_{z z j}^{2}} k_{x 1} k_{x 2}\right) \\
& \gamma_{j}=k_{z L j}^{-} k_{x 1} k_{z L j}^{(2)} \frac{\chi_{x x z j}}{\varepsilon_{x x j}} \\
& +k_{x 3}\left(\frac{\chi_{z x x j}}{\varepsilon_{x x j}} k_{z L j}^{(1)} k_{z L j}^{(2)}-\frac{\chi_{z z z j} \varepsilon_{x x j}}{\varepsilon_{z z j}^{2}} k_{x 1} k_{x 2}\right), \\
& k_{z L j}^{ \pm}=k_{z L j}^{(1)} \pm k_{z L j}^{(2)}, \quad k_{z L j}^{(p)}=\sqrt{\left(k_{p}^{2}-\frac{k_{x p}^{2}}{\varepsilon_{z z j}}\right) \varepsilon_{x x j}}, \\
& p=1,2,3 ; \quad j=1,2 .
\end{aligned}
$$

Here $k_{z L j}^{(p)}$ are the $z$ components of the wave vectors in $j$ th layer at frequencies $\omega_{p}$, respectively; superscript $n$ identifies the period number in the stack. The coefficients $B_{j}^{n \pm}\left(\omega_{1,2}\right)$ are the field amplitudes inside the $j$ th layer of the $n$th period at the incident wave frequencies $\omega_{1}$ and $\omega_{2}$. These coefficients are obtained by imposing the continuity conditions for the tangential field components of each pump wave of frequencies $\omega=\omega_{1,2}$ independently at the layer interfaces and can be represented in the form:

$$
\begin{aligned}
& B_{j}^{n \pm}\left(\omega_{p}\right) \\
& \quad=\left(s_{11 j}^{(n)}\left(\omega_{p}\right) \pm \frac{k_{p}}{k_{z L j}^{(p)}} \varepsilon_{x x j} s_{21 j}^{(n)}\left(\omega_{p}\right)\right)\left(1+R\left(\omega_{p}\right)\right)+\frac{k_{z a}^{(p)}}{k_{p} \varepsilon_{a}}\left(s_{12 j}^{(n)}\left(\omega_{p}\right) \pm \frac{k_{p}}{k_{z L j}^{(p)}} \varepsilon_{x x j} s_{22 j}^{(n)}\left(\omega_{p}\right)\right)\left(1-R\left(\omega_{p}\right)\right), \\
& R\left(\omega_{p}\right) \\
& \quad=\frac{M_{11}\left(\omega_{p}\right)+\left(k_{z a}^{(p)} /\left(\varepsilon_{a} k_{p}\right)\right) M_{12}\left(\omega_{p}\right)-\left(\varepsilon_{a} k_{p} / k_{z a}^{(p)}\right) M_{21}\left(\omega_{p}\right)-M_{22}\left(\omega_{p}\right)}{M_{11}\left(\omega_{p}\right)+\left(k_{z a}^{(p)} /\left(\varepsilon_{a} k_{p}\right)\right) M_{12}\left(\omega_{p}\right)+\left(\varepsilon_{a} k_{p} / k_{z a}^{(p)}\right) M_{21}\left(\omega_{p}\right)+M_{22}\left(\omega_{p}\right)} ; p=1,2,
\end{aligned}
$$


where $k_{z a}^{(p)}=k_{p} \sqrt{\varepsilon_{a}} \cos \Theta_{i p}$ is the longitudinal wavenumber in the surrounding medium and $R\left(\omega_{p}\right)$ is the reflection coefficient at frequency $\omega_{p}$. The transfer matrix $\widehat{M}\left(\omega_{p}\right)$ of the finite linear periodic structure containing $N$ periods can be expressed in terms of the transfer matrix $\hat{m}\left(\omega_{p}\right)=$ $\hat{m}_{L 1}\left(\omega_{p}\right) \hat{m}_{L 2}\left(\omega_{p}\right)$ of a single period using Abeles theorem [21]: $\widehat{M}\left(\omega_{p}\right)=\left(\hat{m}\left(\omega_{p}\right)\right)^{N}$, where $\hat{m}_{L 1, L 2}\left(\omega_{p}\right)$ are the transfer matrices of the constituent layers of the unit cell. The matrices $\hat{s}_{j}^{(n)}$ in (7) are defined as follows: $\hat{s}_{1}^{(n)}\left(\omega_{p}\right)=$ $\left(\hat{m}\left(\omega_{p}\right)^{n-1}\right)^{-1}$ and $\widehat{s}_{2}^{(n)}\left(\omega_{p}\right)=\left(\hat{m}\left(\omega_{p}\right)^{n-1} \cdot \hat{m}_{L 1}\left(\omega_{p}\right)\right)^{-1}$.

To satisfy the boundary conditions at the interfaces of the nonlinear layers at the combinatorial frequency $\omega_{3}$, the TMM procedure has to be modified in order to take into account the contribution of the frequency mixing products generated in each layer and subsequently refracted through the periodic stack. Namely, the fields at interfaces of the first layer in the binary unit cell are related as follows:

$$
\begin{aligned}
\left(\begin{array}{c}
H_{y 1}^{(1)}\left(\omega_{3}, x, 0\right) \\
E_{x 1}^{(1)}\left(\omega_{3}, x, 0\right)
\end{array}\right)= & \hat{m}_{L 1}\left(\omega_{3}\right)\left(\begin{array}{c}
H_{y 1}^{(1)}\left(\omega_{3}, x, d_{1}\right) \\
E_{x 1}^{(1)}\left(\omega_{3}, x, d_{1}\right)
\end{array}\right) \\
& +\hat{m}_{L 1}\left(\omega_{3}\right)\left(\begin{array}{c}
\tau_{11}\left(d_{1}\right) \\
\xi_{11}\left(d_{1}\right)
\end{array}\right) .
\end{aligned}
$$

Similarly, for the second layer we obtain

$$
\begin{aligned}
\left(\begin{array}{c}
H_{y 1}^{(1)}\left(\omega_{3}, x, d_{1}\right) \\
E_{x 1}^{(1)}\left(\omega_{3}, x, d_{1}\right)
\end{array}\right)= & \hat{m}_{L 2}\left(\omega_{3}\right)\left(\begin{array}{c}
H_{y 2}^{(1)}\left(\omega_{3}, x, d_{1}+d_{2}\right) \\
E_{x 2}^{(1)}\left(\omega_{3}, x, d_{1}+d_{2}\right)
\end{array}\right) \\
& +\hat{m}_{L 2}\left(\omega_{3}\right)\left(\begin{array}{l}
\tau_{21}\left(d_{1}+d_{2}\right) \\
\xi_{21}\left(d_{1}+d_{2}\right)
\end{array}\right) .
\end{aligned}
$$

Thus, (8) and (9) define the interrelation between the fields at the external interfaces of the constituent unit cell. After applying the boundary conditions sequentially to all $N$ unit cells, the fields at the stack outer interfaces can be represented in the form:

$$
\begin{aligned}
\left(\begin{array}{c}
H_{y 1}^{(1)}\left(\omega_{3}, x, 0\right) \\
E_{x 1}^{(1)}\left(\omega_{3}, x, 0\right)
\end{array}\right) & \\
= & \widehat{M}\left(\omega_{3}\right)\left(\begin{array}{c}
H_{y 2}^{(N)}\left(\omega_{3}, x, L\right) \\
E_{x 2}^{(N)}\left(\omega_{3}, x, L\right)
\end{array}\right)+\hat{m}_{L 1}\left(\omega_{3}\right)\left(\begin{array}{l}
\tau_{11}\left(d_{1}\right) \\
\xi_{11}\left(d_{1}\right)
\end{array}\right) \\
& +\hat{m}_{L 1}\left(\omega_{3}\right) \hat{m}_{L 2}\left(\omega_{3}\right)\left(\begin{array}{l}
\tau_{21}\left(d_{1}+d_{2}\right) \\
\xi_{21}\left(d_{1}+d_{2}\right)
\end{array}\right) \\
& +\cdots+\widehat{M}\left(\omega_{3}\right)\left(\begin{array}{l}
\tau_{2 N}(L) \\
\xi_{2 N}(L)
\end{array}\right) .
\end{aligned}
$$

Here $\tau_{j n}$ and $\xi_{j n}$ contain the terms proportional to coefficients $D_{1 j, 2 j}^{n \pm}, j=1,2$ :

$$
\begin{gathered}
\tau_{j n}=D_{1 j}^{n+} \sigma_{1 j}^{+}+D_{2 j}^{n+} \sigma_{1 j}^{-}+D_{1 j}^{n-} \sigma_{2 j}^{+}+D_{2 j}^{n-} \sigma_{2 j}^{-}, \\
\xi_{j n}=D_{1 j}^{n+} \sigma_{3 j}^{+}+D_{2 j}^{n+} \sigma_{3 j}^{-}+D_{1 j}^{n-} \sigma_{4 j}^{+}+D_{2 j}^{n-} \sigma_{4 j}^{-}, \\
\sigma_{1 j}^{ \pm}=\cos k_{z L j}^{(3)} d_{j} \pm i \frac{k_{z L j}^{+}}{k_{z L j}^{(3)}} \sin k_{z L j}^{(3)} d_{j}-e^{ \pm i k_{z L j}^{+} d_{j},}, \\
\sigma_{2 j}^{ \pm}=\cos k_{z L j}^{(3)} d_{j} \pm i \frac{k_{z L j}^{-}}{k_{z L j}^{(3)}} \sin k_{z L j}^{(3)} d_{j}-e^{ \pm i k_{z L j}^{-} d_{j}}, \\
\sigma_{3 j}^{ \pm}=\frac{c}{\omega_{3}} \frac{k_{z L j}^{(3)}}{\varepsilon_{x x j}}\left(i \sin k_{z L j}^{(3)} d_{j} \pm \frac{k_{z L j}^{+}}{k_{z L j}^{(3)}} \cos k_{z L j}^{(3)} d_{j} \mp \frac{k_{z L j}^{+}}{k_{z L j}^{(3)}} e^{ \pm i k_{z L j}^{+} d_{j}}\right), \\
\sigma_{4 j}^{ \pm}=\frac{c}{\omega_{3}} \frac{k_{z L j}^{(3)}}{\varepsilon_{x x j}}\left(i \sin k_{z L j}^{(3)} d_{j} \pm \frac{k_{z L j}^{-}}{k_{z L j}^{(3)}} \cos k_{z L j}^{(3)} d_{j} \mp \frac{k_{z L j}^{-}}{k_{z L j}^{(3)}} e^{ \pm i k_{z L j}^{+} d_{j}}\right) .
\end{gathered}
$$

The magnetic field of frequency $\omega_{3}$ emitted from the stack of nonlinear layers into the surrounding homogeneous medium has the form:

$$
H_{y}^{a}\left(\omega_{3}\right)=e^{-i \omega_{3} t+i k_{x 3} x} \begin{cases}F_{r} e^{-i k_{2 a}^{(3)} z}, & z \leq 0, \\ F_{t} e^{i k_{2 a}^{(3)} z}, & z \geq L,\end{cases}
$$

where $k_{z a}^{(3)}=\sqrt{k_{3}^{2} \varepsilon_{a}-k_{x 3}^{2}}$ is the longitudinal wave number of the wave at frequency $\omega_{3}$ in the homogeneous media and the nonlinear scattering coefficient $F_{r}$ and $F_{t}$ are determined by enforcing the interface boundary conditions at $z=0, L$.

Finally, by combining (5), (10), and (12) we obtain the sought coefficients $F_{r, t}$ :

$$
\begin{gathered}
F_{r}=\left(\frac{k_{3} \varepsilon_{a}}{k_{z a}^{(3)}}\left(\hat{\eta}_{N}\right)_{21}+\left(\hat{\eta}_{N}\right)_{22}\right) \lambda_{1}-\left(\frac{k_{3} \varepsilon_{a}}{k_{z a}^{(3)}}\left(\hat{\eta}_{N}\right)_{11}+\left(\hat{\eta}_{N}\right)_{12}\right) \lambda_{2}, \\
F_{t}=-\left(\lambda_{1}+\lambda_{2} \frac{k_{3} \varepsilon_{a}}{k_{z a}^{(3)}}\right),
\end{gathered}
$$

where

$$
\begin{aligned}
& \lambda_{p}=\frac{1}{\Delta} \sum_{n=1}^{N}\left[\left(\hat{\eta}_{n}^{\prime}\right)_{p 1} \tau_{1 n}+\left(\hat{\eta}_{n}^{\prime}\right)_{p 2} \xi_{1 n}+\left(\hat{\eta}_{n}\right)_{p 1} \tau_{2 n}+\left(\hat{\eta}_{n}\right)_{p 2} \xi_{2 n}\right], \\
& p=1,2 ; \\
& \Delta=\left(\hat{\eta}_{N}\right)_{11}+\left(\hat{\eta}_{N}\right)_{22}+\frac{k_{z a}^{(3)}}{k_{3} \varepsilon_{a}}\left(\hat{\eta}_{N}\right)_{12}+\frac{k_{3} \varepsilon_{a}}{k_{z a}^{(3)}}\left(\hat{\eta}_{N}\right)_{21} ; \\
& \hat{\eta}_{n}=\left[\hat{m}\left(\omega_{3}\right)\right]^{n}, \quad \hat{\eta}_{n}^{\prime}=\hat{\eta}_{n-1} \hat{m}_{L 1}\left(\omega_{3}\right), \quad \hat{\eta}_{N}=\widehat{M}\left(\omega_{3}\right) .
\end{aligned}
$$

It is necessary to note that $F_{r, t}$ in (13) always remain finite inspite of the fact that coefficients $D_{1 j, 2 j}^{n \pm}$ have singularity at $k_{z L j}^{ \pm}=k_{z L j}^{(3)}$. However, it can be shown that coefficients 
$A_{j}^{n \pm}$ in (5) contain exactly the same pole as $D_{1 j, 2 j}^{n \pm}$ at $\Theta_{i 1}=\Theta_{i 2}$, and their combined contribution is finite at all frequencies and incidence angles.

Thus the modified TMM approach presented in this section gives the closed-form expressions for the nonlinear scattering coefficients of the finite $\mathrm{PhC}$ composed of the binary nonlinear layers. The obtained analytical formulations not only provide a qualitative insight in the formation of the nonlinear response and the properties of the scattered fields but also enable fast quantitative analysis of the specific $\mathrm{PhC}$ configurations.

The results of numerical simulations based upon the analytical solutions obtained here are presented in the next section to illustrate the effects of structure and materials parameters on the properties TM waves of combinatorial frequencies generated by nonlinear $\mathrm{PhC}$ in the three-wave mixing process.

\section{Properties and Mechanisms of Nonlinear Scattering by Finite Periodic Stacks}

The analytical solutions for the coefficients $F_{r, t}$ obtained in the preceding section have allowed us to examine the mechanisms of nonlinear scattering in 1D anisotropic nonlinear PhCs. The effects of the constituent layer parameters, unit cell aspect ratio, and the pump wave frequencies $\omega_{1,2}$ and incidence angles $\Theta_{i 1,2}$ on the properties of the waves of combinatorial frequency $\omega_{3}=\omega_{1}+\omega_{2}$ generated in the three-wave mixing process have been analysed with the aim of increasing the efficiency of nonlinear processes in the artificial medium.

To illustrate the features of the frequency mixing in the 1D nonlinear anisotropic PhCs, the characteristics of the combinatorial frequency waves are discussed here with the examples of periodic stacks of binary anisotropic dielectric layers of $\mathrm{CdS}$ and $\mathrm{ZnO}$ described by the tensors $\hat{\varepsilon}$ and $\hat{\chi}(1)$ with the following parameters [22]:

CdS: $\varepsilon x x 1=5.382, \varepsilon_{z z 1}=5.457\left(\alpha_{1}=\varepsilon_{x x 1} / \varepsilon_{z z 1}=\right.$ $0.986), \chi_{x x z 1}=2.1 \times 10^{-7}, \chi_{z x x 1}=1.92 \times 10^{-7}, \chi_{z z z 1}=$ $3.78 \times 10^{-7}$

$\mathrm{ZnO}: \varepsilon_{x x 2}=1.4, \varepsilon_{z z 2}=2.6\left(\alpha_{2}=\varepsilon_{x x 2} / \varepsilon_{z z 2}=0.538\right)$, $\chi_{x x z 2}=2.82 \times 10^{-8}, \chi_{z x x 2}=2.58 \times 10^{-8}, \chi_{z z z 2}=8.58 \times$ $10^{-8}$.

The constituent layer thicknesses are $d_{1}=0.08 \mathrm{~mm}$ and $d_{2}=0.05 \mathrm{~mm}$, unless specifically defined. Exterior of the layer stack in Figure 1 is an air with permittivity $\varepsilon_{a}=1$.

\subsection{Spectral Efficiency of the Combinatorial Frequency Gen-} eration. PhCs are known to be instrumental in enhancing the SHG and THG efficiency by choosing the pump wave frequency close to the $\mathrm{PhC}$ band edge. Therefore, it was interesting to explore whether similar facility could be exploited for the combinatorial frequencies generated in the three-wave mixing process. The spectral bands of a periodic stack of binary linear anisotropic dielectric layers have been inferred first from the reflectance $|R(\omega)|$ of the pump waves. Figure 2 illustrates $|R(\omega)|$ for the TM wave incident at angle

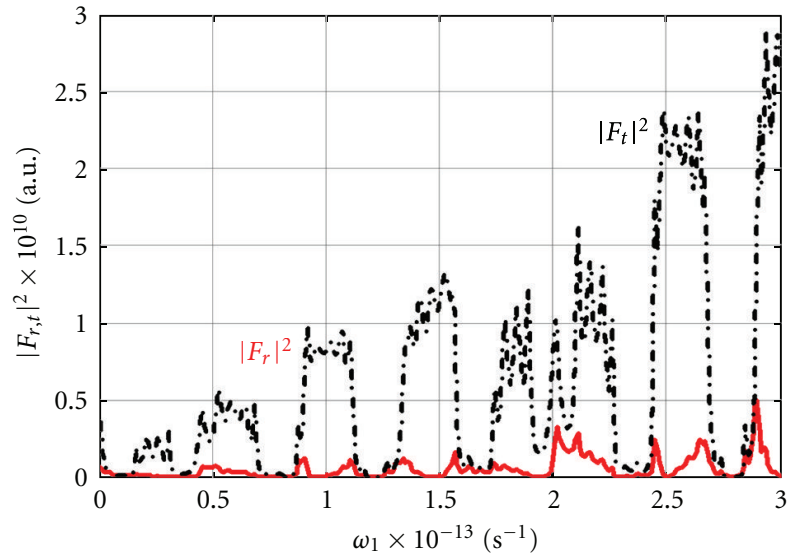

Figure 2: Reflectance of plane TM wave incident at $\Theta_{i}=30^{\circ}$ on the periodic stack of $N=7$ binary dielectric layers of thicknesses $d_{1}=0.08 \mathrm{~mm}$ and $d_{2}=0.05 \mathrm{~mm}$.

$\Theta_{i 1}=30^{\circ}$ on the periodic stack containing $N=7$ unit cells. The bandgaps, corresponding to $|R(\omega)| \approx 1$, are clearly observable in Figure 2, but it is necessary to note that the respective frequency bands change with the incidence angle and layers' parameters.

The field intensities $\left|F_{r, t}\right|^{2}$ at the combinatorial frequency $\omega_{3}=\omega_{1}+\omega_{2}$ generated in the same structure are shown in Figure 3 for variable frequency $\omega_{1}$ of a pump wave incident at $\Theta_{i 1}=30^{\circ}$, while the frequency $\omega_{2}=1.135 \times 10^{13} \mathrm{~s}^{-1}$ of the other pump wave, incident at $\Theta_{i 2}=45^{\circ}$, was fixed at the passband edge. Comparison of Figures 2 and 3 demonstrates strong correlation between $\left|F_{r, t}\right|^{2}$ and $|R(\omega)|$. However, in contrast to SHG and THG, the band edges have little effect on the $\omega_{3}$ generation efficiency, namely, $\left|F_{t}\right|^{2}$ reaches its maxima inside the transparency bands, and only $\left|F_{r}\right|^{2}$ exhibits small kinks at the band edges when frequency $\omega_{1}$ of the first pump wave varies.

Figure 3 also shows that the peak intensity $\left|F_{t}\right|^{2}$ grows with $\omega_{1}$ and the efficiency of the frequency conversion is higher when the $\omega_{1}$ remains inside the pump wave transparency bands. This effect can be attributed to the increase of the pump wave interaction length at the higher frequencies further assisted by the enhanced mixing efficiency at Wolf-Bragg resonances of Bloch waves in the finite PhCs. It is noteworthy that $(N-1)$ resonances occur in each transparency band of the $N$-cell stack. At these resonances $|R(\omega)|=0$ as the stack overall thickness equals an integer number of Bloch half-waves with the wavenumbers $\bar{k}(\omega)$, that is, $N \bar{k}(\omega)\left(d_{1}+d_{2}\right)=\pi q, q=0, \pm 1, \pm 2, \ldots$, where $\bar{k}(\omega)$ is defined by the relation $\cos \bar{k}\left(d_{1}+d_{2}\right)=\left(m_{11}+m_{22}\right) / 2, m_{11}$ and $m_{22}$ are elements of the unit cell transfer matrix $\hat{m}(\omega)$ defined in connection with (7).

3.2. Effect of the Stack Thickness. As indicated in the preceding section, the number $N$ of stacked unit cells and thickness of the whole stack may have strong impact on the efficiency of harmonic generation in nonlinear PhC. This effect has been predicted by the analytical formulations (13) and 


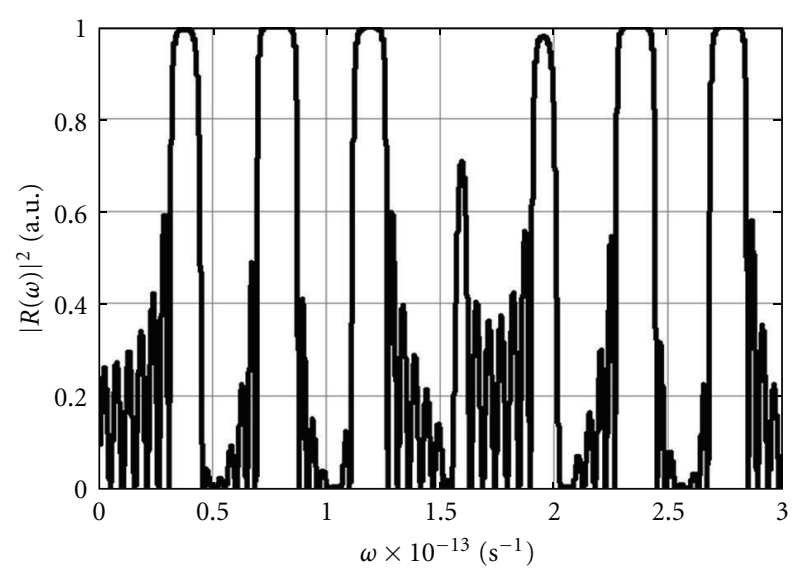

Figure 3: The field intensity at frequency $\omega_{3}=\omega_{1}+\omega_{2}$ radiated in the reverse $\left(\left|F_{r}\right|^{2}\right.$ : red solid line) and forward $\left(\left|F_{t}\right|^{2}\right.$ : black dashdot line) directions of the $z$-axis at $\Theta_{i 1}=30^{\circ} ; \Theta_{i 2}=45^{\circ}, N=7$, $d_{1}=0.08 \mathrm{~mm}, d_{2}=0.05 \mathrm{~mm}$, and $\omega_{2}=1.135 \times 10^{13} \mathrm{~s}^{-1}$.

confirmed by the numerical simulations in Figure 4. Indeed, the field intensity $\left|F_{r, t}\right|^{2}$ exhibits nonmonotonic dependences on the number $N$ of unit cells in the stack as illustrated by Figure 4 for two different combinatorial frequencies $\omega_{3}=$ $\omega_{1}+\omega_{2}$ (the pump wave frequencies $\omega_{1}$ and $\omega_{2}$ are close to the $\mathrm{PhC}$ band edges in both cases). Indeed, Figure 4(a) shows that $\left|F_{r}\right|^{2}$ has maxima at $N=32,57,89, \ldots$, whereas $\left|F_{t}\right|^{2}$ has a higher peak at $N=32$ and then follows almost the same pattern as $\left|F_{r}\right|^{2}$. However, at the higher frequency $\omega_{1}$, maxima of $\left|F_{r, t}\right|^{2}$ occur at $N=108$ and $N=127$ as shown in Figure 4(b), where the peak values of $\left|F_{t}\right|^{2}$ are about two orders of magnitude higher than those in Figure 4(a) and about 20 times larger than for $\left|F_{r}\right|^{2}$. The $\left|F_{r, t}\right|^{2}$ can also exhibit giant growth and reach their extrema at Wolf-Bragg resonances of very high orders in rather thick stacks with the special combinations of the pump wave frequencies, incidence angles, and the layer parameters as suggested in [13].

3.3. Effect of the Pump Wave Incidence Angles on the Frequency Mixing Efficiency. Harmonic generation in 1D PhCs are usually analysed at normal incidence of pump wave on the stacked layers. In the case of combinatorial frequency generation by a pair of pump waves, incident at different angles, an additional degree of freedom exists in realising the phase synchronism and controlling the whole frequency mixing process. To gain insight in the effect of the incidence angle on the combinatorial frequency field intensities, $\left|F_{r, t}\right|^{2}$ have been simulated at variable incidence angle $\Theta_{i 1}$ and fixed angle $\Theta_{i 2}$ of the respective pump waves and different number of the unit cells in the stack: $N=7,15,25$.

Examination of $\left|F_{r, t}\left(\Theta_{i 1}\right)\right|^{2}$ in Figure 5 shows that when the stack is relatively thin $(N=7)$, both $\left|F_{r}\right|^{2}$ and $\left|F_{t}\right|^{2}$ exhibit similar behaviour and smoothly vary with $\Theta_{i 1}$. However, additional resonances arise in the thicker stacks, and the $\left|F_{r, t}\left(\Theta_{i 1}\right)\right|^{2}$ dependencies qualitatively change. Several factors are responsible for these alterations. At first, dissimilar reflectance and transmittance of the individual pump waves have significant effect on the ratio of the pump wave amplitudes in the three-wave mixing process. Secondly, angular variations of the $\mathrm{PhC}$ transparency bands become more noticeable in the thicker stacks. Finally, the higher order spatial harmonics, which can resonate in thicker stacks, contribute to the combinatorial frequency generation.

Both the reflectance/transmittance of pump waves and the phase synchronism in the mixing process are essentially dependent on the permittivities and anisotropy of the constituent binary layers. Therefore the effect of the constituent layer parameters has been assessed first to discriminate contributions of the aforementioned mechanisms to the combinatorial frequency generation. In order to evaluate the effect of the layer anisotropy, the intensities $\left|F_{t}\left(\Theta_{i 1}\right)\right|^{2}$ have been simulated at the modified permittivity ratios $\varepsilon_{x x 1} / \varepsilon_{z z 1}=2 \alpha_{1}, \varepsilon_{x x 2} / \varepsilon_{z z 2}=2 \alpha_{2}$, and $\varepsilon_{x x 1} / \varepsilon_{z z 1}=\alpha_{1} / 2$, $\varepsilon_{x x 2} / \varepsilon_{z z 2}=\alpha_{2} / 2$ and are shown in Figure 6. Comparison of the plots in Figure 5(b) for $\varepsilon_{x x 1} / \varepsilon_{z z 1}=\alpha_{1}, \varepsilon_{x x 2} / \varepsilon_{z z 2}=$ $\alpha_{2}$ with the respective plots in Figure 6 for the modified tensor $\widehat{\varepsilon}$ demonstrates that variations of the layer anisotropy qualitatively alter the efficiency of the combinatorial frequency generation. Namely, we can observe that when the layer anisotropy deviates from the specified values of $\alpha_{1,2}$ in either direction, the combinatorial frequency intensity considerably decreases, from a few times to several orders of magnitude. Furthermore, additional angular undulations of the field intensity occur at several incidence angles, Figure 6, being inflicted by the resonances of the higher order spatial harmonics.

\subsection{Effects of Constituent Layer Thicknesses and Resonance} Enhancement of Frequency Conversion. The stack overall thickness may have profound influence on the frequency mixing efficiency. This can be the result of the increased number of unit cells in the stack as illustrated in Figure 4 or variations in the thicknesses of the constituent layers. The earlier studies have demonstrated that the efficiency of combinatorial frequency generation can significantly vary with thickness of an individual nonlinear layer at the higher order Wolf-Bragg resonances [13]. This suggests that the aspect ratio of the binary layers in the unit cell as well as the unit cell size can provide independent controls of the dispersion and the pump wave reflectance/transmittance. In order to elucidate this effect, the intensities $\left|F_{r, t}\right|^{2}$ at frequency $\omega_{3}=\omega_{1}+\omega_{2}$ have been analysed at the variable thickness of one layer, while thickness of the other was fixed. Figure 7 displays $\left|F_{r, t}\right|^{2}$ for a stack with $N=7$ unit cells illuminated by the pump waves incident at $\Theta_{i 1}=38^{\circ}$ and $\Theta_{i 2}=45^{\circ}$ corresponding to the maximal intensity of $\left|F_{r, t}\right|^{2}$ for the reference unit cell with $d_{1}=0.08 \mathrm{~mm}$ and $d_{2}=0.05 \mathrm{~mm}$ in Figure 5. It can be seen that both $\left|F_{r, t}\right|^{2}$ grow with thickness of the layers in the period, while $\left|F_{t}\right|^{2}$ always remains greater than $\left|F_{r}\right|^{2}$. It is necessary to note that the growth rate of the $\left|F_{r, t}\right|^{2}$ versus $d_{1}$ (Figure $\left.7(\mathrm{~b})\right)$ is higher than that versus $d_{2}$ (Figure $7(\mathrm{a})$ ) for nearly an order of magnitude. This effect is directly related to the fact that the components of the nonlinear susceptibility tensor $\hat{\chi}$ in 


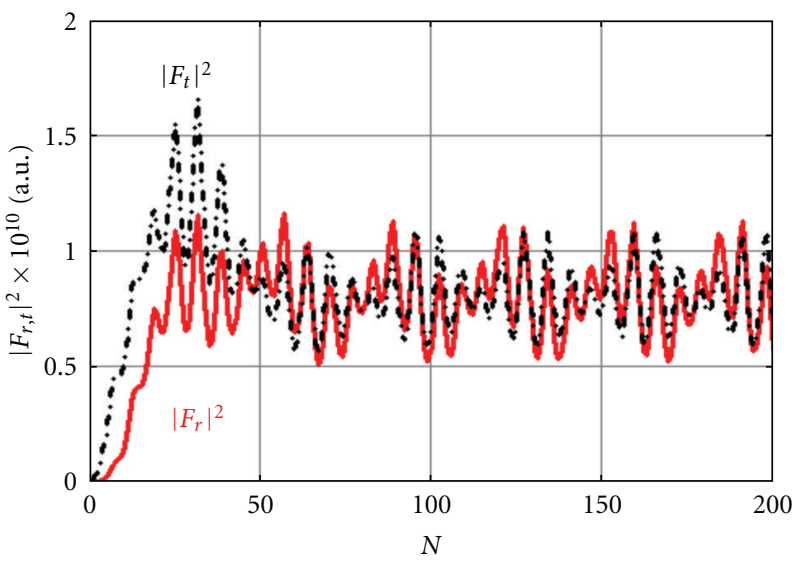

(a)

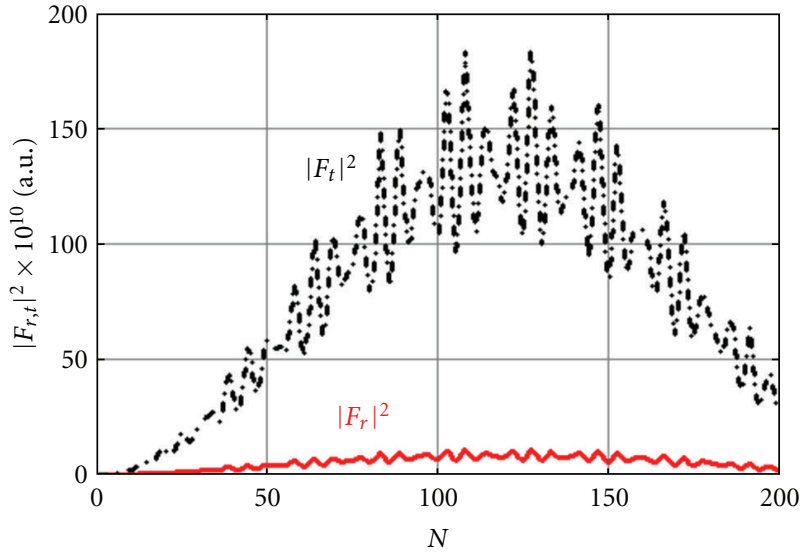

(b)

FIGURE 4: Intensities $\left|F_{r, t}\right|^{2}$ of the field at frequency $\omega_{3}=\omega_{1}+\omega_{2}$ radiated in the reverse $\left(\left|F_{r}\right|^{2}\right.$ : red solid line) and forward $\left(\left|F_{t}\right|^{2}\right.$ : black dash-dot line) directions of the $z$-axis at $\Theta_{i 1}=30^{\circ}, \Theta_{i 2}=45^{\circ}, \omega_{2}=1.135 \times 10^{13} \mathrm{~s}^{-1}$ and (a) $\omega_{1}=0.4985 \times 10^{13} \mathrm{~s}^{-1} ;(\mathrm{b}) \omega_{1}=1.55 \times 10^{13} \mathrm{~s}^{-1}$.

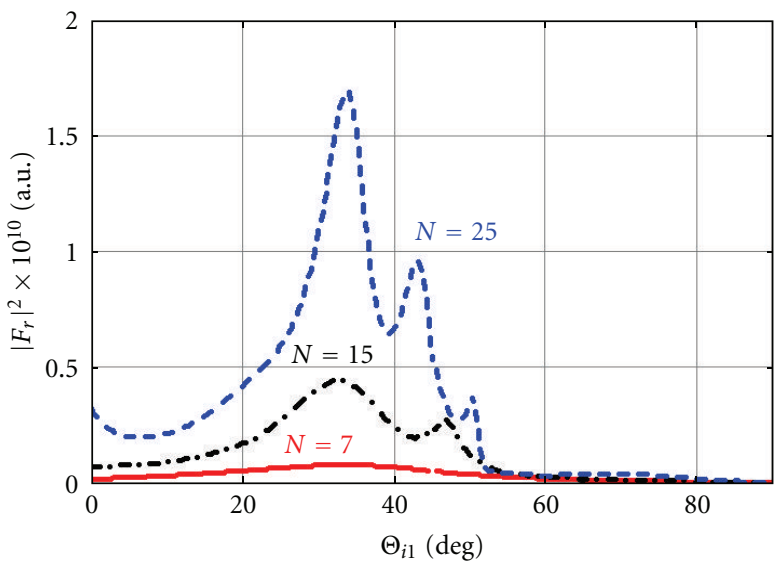

(a)

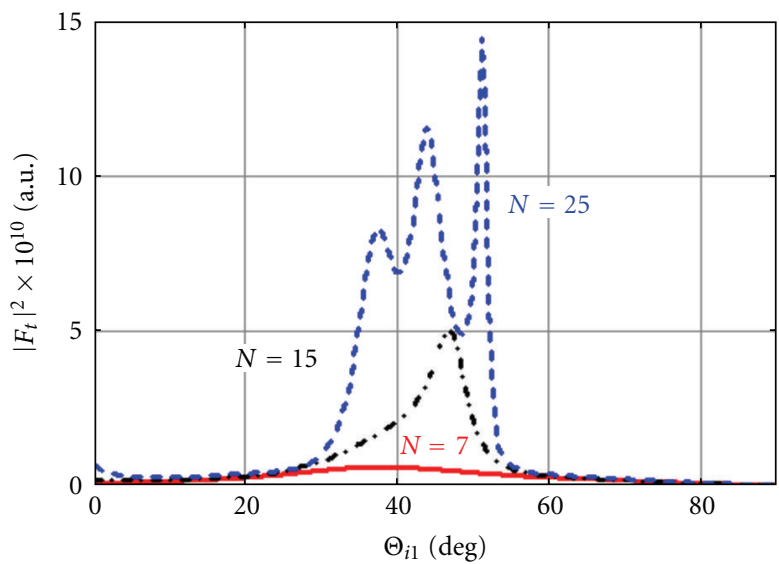

(b)

FIGURE 5: The field intensity at frequency $\omega_{3}=\omega_{1}+\omega_{2}$ radiated in the reverse (a) and forward (b) directions of the $z$-axis; pump waves of frequencies $\omega_{1}=0.4585 \times 10^{13} \mathrm{~s}^{-1}$ and $\omega_{2}=1.135 \times 10^{13} \mathrm{~s}^{-1}$ are incident at $\Theta_{i 2}=45^{\circ}$ and variable angle $\Theta_{i 1}$.

the first layer are an order of magnitude greater than in the second one. Therefore when both layers are thin as compared with the wavelength, $\left|F_{r, t}\right|^{2}$ are small at the low order WolfBragg resonances. However, when thickness of one of the layers increases, this leads to considerable difference in the $\left|F_{r, t}\right|^{2}$ undulation frequencies and the growth rates due to substantial dissimilarity of the constitutive parameters of the layers in the unit cell. The additional periodic undulations of $\left|F_{r, t}\right|^{2}$ can also be attributed to unequal variations of the reflection coefficients $R\left(\omega_{1,2}\right)$ of the incident pump waves which cause the pump wave amplitude and phase disbalance in the mixing process.

The analytical study of nonlinear scattering by an isolated anisotropic dielectric slab in [13] has revealed that the efficiency of the combinatorial frequency generation can be increased for several orders of magnitude at the high-order
Wolf-Bragg resonances. In particular, in the CdS slab with the parameters $\hat{\varepsilon}_{1}$ and $\hat{\chi}_{1}$ defined at the beginning of this section, $\left|F_{r, t}\right|^{2}$ reaches the global maxima at the thickness $d_{1}=$ $1.695 \mathrm{~mm}$, frequency ratio $\omega_{1} / \omega_{2}=1.911$, the pump wave incidence angles $\Theta_{i 1}=30^{\circ}, \Theta_{i 2}=60^{\circ}$. Similar analysis has been performed here for the periodic stacks containing $N=$ 7 unit cells with the thick binary layers of $\mathrm{CdS}$ and $\mathrm{ZnO}$. The simulation results in Figure 8 shows that the higher order Wolf-Bragg resonances in individual layers create additional modulation of the $\left|F_{r, t}\right|^{2}$ magnitude and the peak intensity is reached at $d_{2}=1.35 \mathrm{~mm}, 4.05 \mathrm{~mm}$ and multiples of these thicknesses. In contrast to the case of an isolated layer, there is no full cancellation of the combinatorial frequency generation at the Wolf-Bragg resonances in the layer 2 . Therefore when $d_{2}$ changes, $\left|F_{r, t}\right|^{2}$ vary about the same median level determined by the layer of thickness $d_{1}$. 


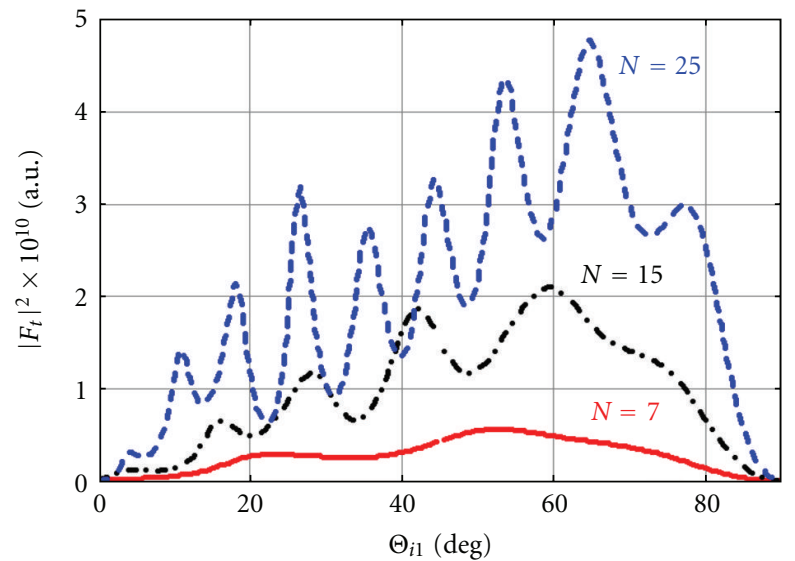

(a)

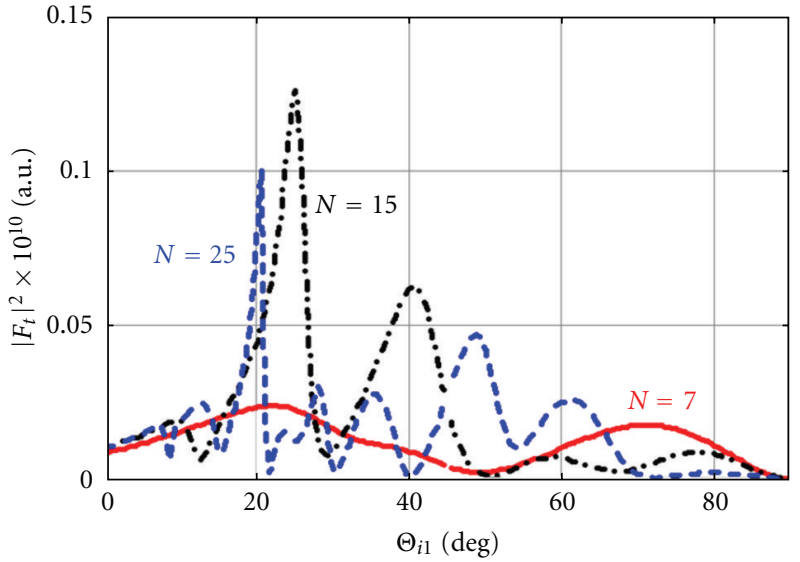

(b)

Figure 6: The field intensity at frequency $\omega_{3}=\omega_{1}+\omega_{2}$ radiated in the forward direction of the $z$-axis; pump waves of frequencies $\omega_{1}=$ $0.4585 \times 10^{13} \mathrm{~s}^{-1}$ and $\omega_{2}=1.135 \times 10^{13} \mathrm{~s}^{-1}$ are incident at $\Theta_{i 2}=45^{\circ}$ and variable angle $\Theta_{i 1}$; the constituent binary layers have the modified anisotropy (a) $\varepsilon_{x x 1} / \varepsilon_{z z 1}=2 \alpha_{1}$ and $\varepsilon_{x x 2} / \varepsilon_{z z 2}=2 \alpha_{2}$; (b) $\varepsilon_{x x 1} / \varepsilon_{z z 1}=\alpha_{1} / 2$ and $\varepsilon_{x x 2} / \varepsilon_{z z 2}=\alpha_{2} / 2$.

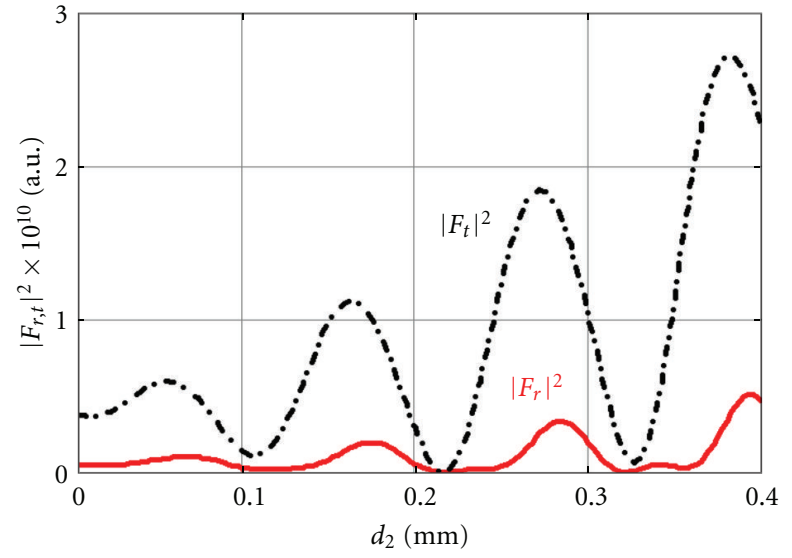

(a)

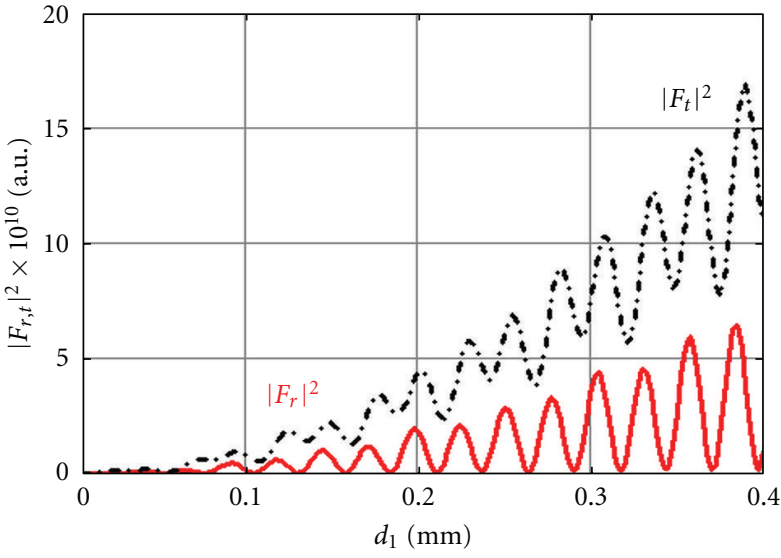

(b)

Figure 7: Intensities $\left|F_{r, t}\right|^{2}$ of the field at frequency $\omega_{3}=\omega_{1}+\omega_{2}$ and the pump waves of frequencies $\omega_{1}=0.4585 \times 10^{13} \mathrm{~s}^{-1}$ and $\omega_{2}=$ $1.135 \times 10^{13} \mathrm{~s}^{-1} ; \Theta_{i 1}=38^{\circ}, \Theta_{i 2}=45^{\circ}, N=7 ;$ (a) $d_{1}=0.08 \mathrm{~mm}$; (b) $d_{2}=0.05 \mathrm{~mm}$.

3.5. Effect of Loss on the Three-Wave Mixing Process in the Periodic Stacks of Binary Layers. The combinatorial frequency generation in the periodic stacks of binary layers discussed so far has been based upon the analysis of the lossless structures. To estimate the effect of dissipation on $\left|F_{r, t}\right|^{2}$, the structures with the same parameters as in Figures 2 and 3 have been simulated in the cases of imperfect layers with the loss tangents $\operatorname{tg} \delta_{x x, z z}=0.01,0.1$. Comparison of the plots in Figures 9 and 10 with the respective results for the lossless cases in Figures 2 and 3 shows that dissipation strongly affects both the reflection coefficients $R\left(\omega_{1,2}\right)$ of the pump waves and the intensities of $\left|F_{r, t}\right|^{2}$ of the generated combinatorial frequencies. First of all, this effect is caused by the lower reflection coefficients $R\left(\omega_{1,2}\right)$ of the pump waves (cf. Figures 2 and 9). In contrast to the lossless case in Figure 3, unequal dissipation of the pump waves in the nonlinear layers of the periodic structure entails an additional disbalance in the three-wave mixing process which further reduces the efficiency of the combinatorial frequency generation, as seen in Figure 10. Moreover, in the case of higher losses $\left(\operatorname{tg} \delta_{x x, z z}=0.1\right.$, Figure $\left.10(\mathrm{~b})\right)$, the combined effect of the pump wave dissipation and attenuation of the mixing products passing through the stack causes dramatic reduction of the $\left|F_{t}\right|^{2}$ peak values, which become nearly an order of magnitude smaller than $\left|F_{r}\right|^{2}$.

The effect of loss on the intensity $\left|F_{r, t}\left(\omega_{3}\right)\right|^{2}$ of the combinatorial frequency generation in the stack of thick layers with variable thickness is illustrated in Figure 11 for the structure with the same parameters as in Figure 8. Comparison of Figures 8 and 11 shows that in the presence of loss, both the median and the first peak levels of the $\left|F_{r, t}\left(\omega_{3}\right)\right|^{2}$ decrease for about 3 times for $\left|F_{r}\right|^{2}$ and 5 times for $\left|F_{t}\right|^{2}$. When thickness of the second layer increases the higher order resonance peaks of $\left|F_{t}\right|^{2}$ progressively decay 


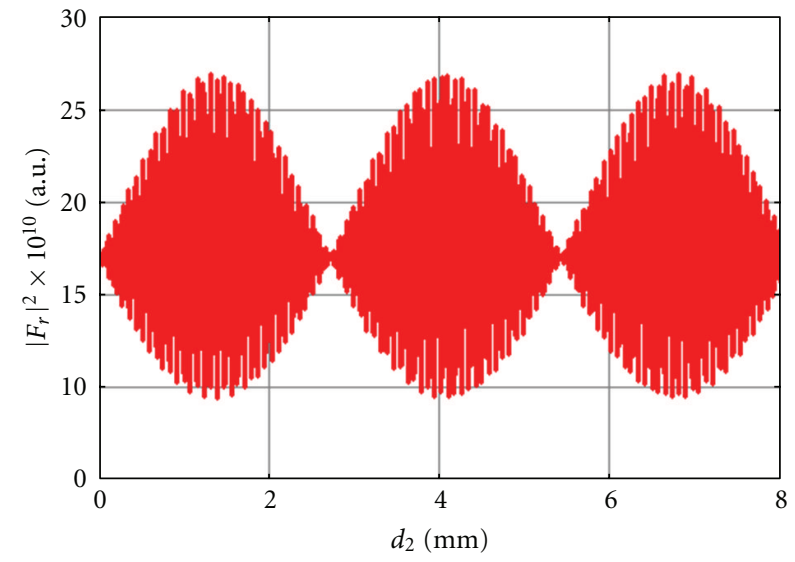

(a)

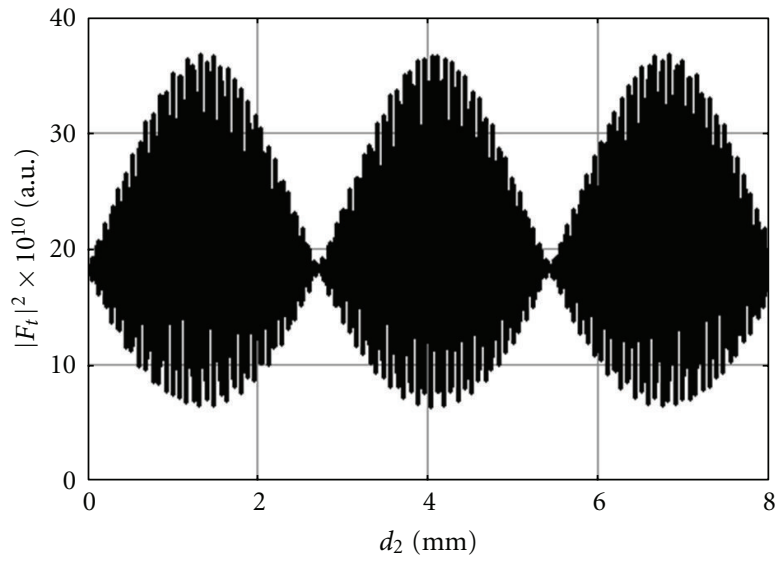

(b)

FIgURE 8: Intensities $\left|F_{r, t}\right|^{2}$ of the field at frequency $\omega_{3}=\omega_{1}+\omega_{2}$ radiated from the stack of the layers in the reverse (a) and forward (b) directions of the $z$-axis at $N=7, d_{1}=1.695 \mathrm{~mm}, \Theta_{i 1}=30^{\circ}, \Theta_{i 2}=60^{\circ}$ and $\omega_{1}=4.982 \times 10^{13} \mathrm{~s}^{-1}, \omega_{2}=2.607 \times 10^{13} \mathrm{~s}^{-1}$.

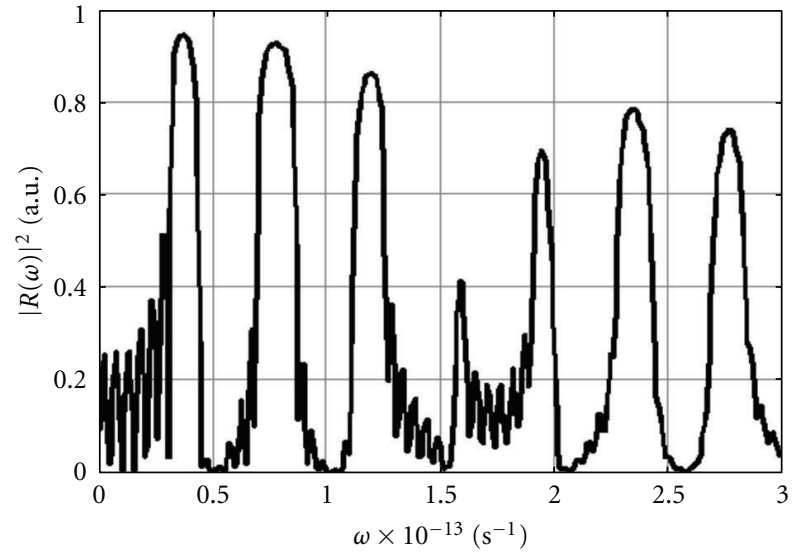

(a)

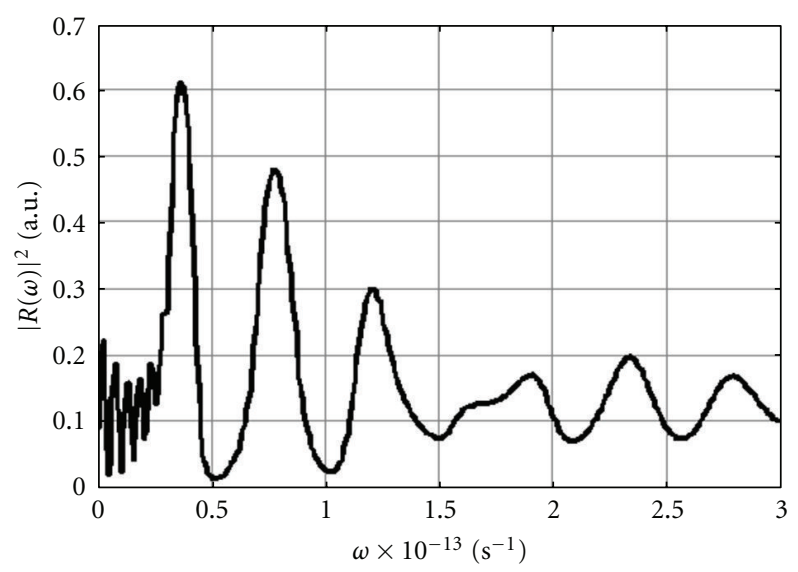

(b)

FIGURE 9: Reflectance of TM wave incident at $\Theta_{i}=30^{\circ}$ on the periodic stack containing $N=7$ unit cells with the dielectric layers of thicknesses $d_{1}=0.08 \mathrm{~mm}, d_{2}=0.05 \mathrm{~mm}$ and (a) $\operatorname{tg} \delta_{x x, z z}=0.01$, (b) $\operatorname{tg} \delta_{x x}, z z=0.1$.

faster than the peak values of $\left|F_{r}\right|^{2}$ as evidenced by Figure 11 . These results show that the strong enhancement of the combinatorial frequency generation efficiency at the high order Wolf-Bragg resonances is feasible at the practical level of dissipation loss.

\section{Conclusions}

The properties and mechanisms of the combinatorial frequency generation by periodic stacks of binary nonlinear anisotropic dielectric layers have been analysed. The closedform solutions for the nonlinear scattering coefficients have been obtained in the approximation of the threewave mixing process in the presence of weak polarisation nonlinearity. The effects of the structure parameters and the incident pump wave characteristics on the efficiency of the combinatorial frequency generation have been investigated in detail. The performed parametric study has shown that in contrast to SHG and THG in the PhCs, the spectral band edges of the binary layer stacks do not improve the combinatorial frequency generation efficiency for the refracted waves. Alternatively, it is shown that the frequency conversion efficiency can be significantly enhanced at WolfBragg resonances occurring at the appropriate combinations of the pump wave frequencies, incidence angles, and the layers' constitutive parameters. The effects of the individual parameters on the frequency mixing efficiency have been discussed in detail for the lossless and lossy constitutive layers in the periodic stacks. It has been demonstrated that the combinatorial frequency generation efficiency can be dramatically increased at the higher order Wolf-Bragg 


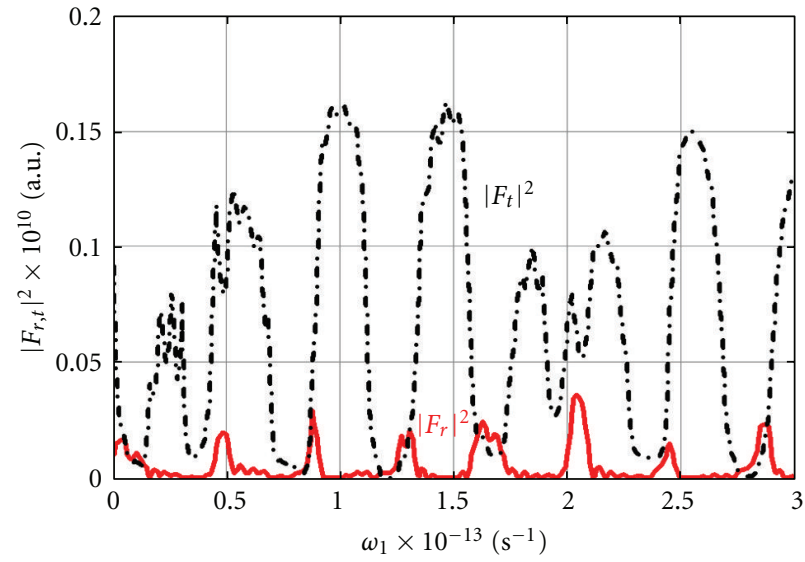

(a)

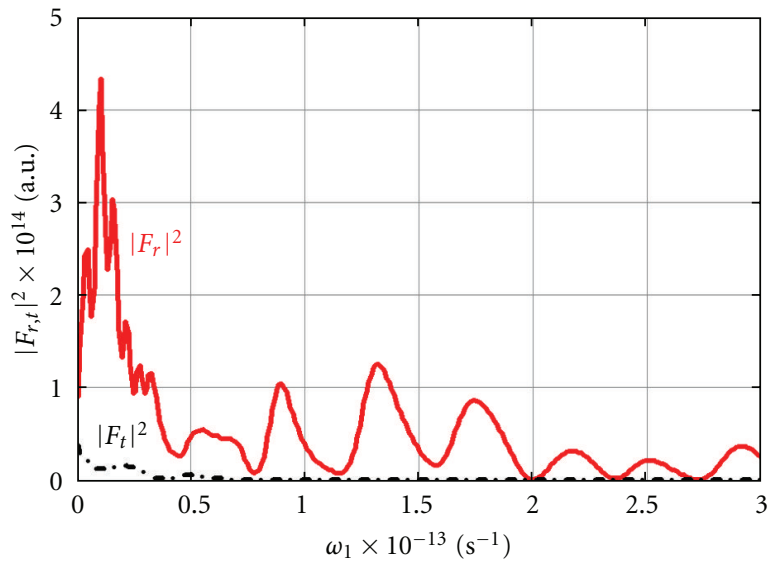

(b)

FIGURE 10: The field intensity at frequency $\omega_{3}=\omega_{1}+\omega_{2}$ radiated in the reverse $\left(\left|F_{r}\right|^{2}\right.$ : red solid line) and forward $\left(\left|F_{t}\right|^{2}\right.$ : black dash-dot line) directions of the $z$-axis at $\Theta_{i 1}=30^{\circ} ; \Theta_{i 2}=45^{\circ}, N=7, d_{1}=0.08 \mathrm{~mm}, d_{2}=0.05 \mathrm{~mm}, \omega_{2}=1.135 \times 10^{13} \mathrm{~s}^{-1}$ and (a) tg $\delta_{x x, z z}=0.01,(\mathrm{~b})$ $\operatorname{tg} \delta_{x x, z z}=0.1$.

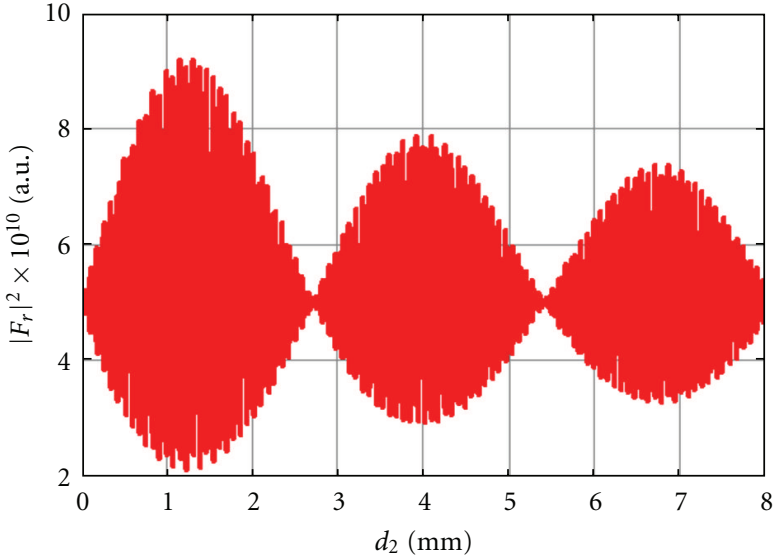

(a)

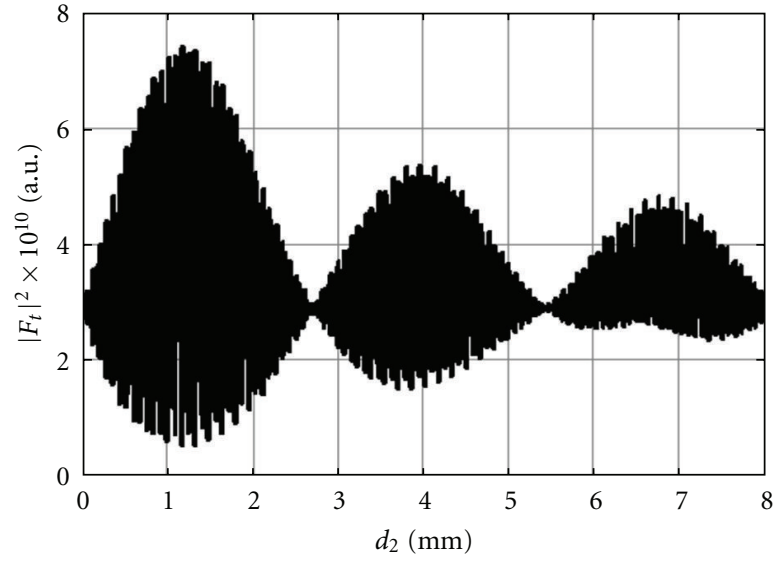

(b)

FIGURE 11: Intensities $\left|F_{r, t}\right|^{2}$ of the field at frequency $\omega_{3}=\omega_{1}+\omega_{2}$ radiated from the periodic stack with $N=7$ unit cells toward the reverse (a) and forward (b) directions of the $z$-axis at $\Theta_{i 1}=30^{\circ}, \Theta_{i 2}=60^{\circ}$ and $\omega_{1}=4.982 \times 10^{13} \mathrm{~s}^{-1}, \omega_{2}=2.607 \times 10^{13} \mathrm{~s}^{-1}, \operatorname{tg} \delta_{x x, z z}=0.001$.

resonances in the stacks with thick constitutive layers. The performed analysis provides insight in the main features of the combinatorial frequency generation by the periodic stacks of binary nonlinear anisotropic dielectric layers.

\section{Acknowledgment}

This work has been performed in the framework of the Project PEARL supported by the FP7 Marie Curie IIF Grant 255110.

\section{References}

[1] M. Centini, C. Sibilia, M. Scalora et al., "Dispersive properties of finite, one-dimensional photonic band gap structures: applications to nonlinear quadratic interactions," Physical Review E, vol. 60, no. 4, pp. 4891-4898, 1999.
[2] Y. Jeong and B. Lee, "Matrix analysis for layered quasi-phasematched media monsidering multiple reflection and pump wave depletion," IEEE Journal of Quantum Electronics, vol. 35, no. 2, pp. 162-172, 1999.

[3] X. Luo and T. Ishihara, "Mode-coexistent phase match condition for second harmonic generation in photonic crystal slabs consisting of centrosymmetric materials," Optics Communications, vol. 242, no. 1-3, pp. 147-154, 2004.

[4] T. V. Murzina, F. Y. Sychev, E. M. Kim et al., "One-dimensional photonic crystals based on porous n -type silicon," Journal of Applied Physics, vol. 98, no. 12, Article ID 123702, pp. 1-4, 2005.

[5] M.-L. Ren and Z. Y. Li, "Exact iterative solution of second harmonic generation in quasi-phase-matched structures," Optics Express, vol. 18, no. 7, pp. 7288-7299, 2010.

[6] J.-J. Li, Z. Y. Li, and D. Z. Zhang, "Second harmonic generation in one-dimensional nonlinear photonic crystals solved by the transfer matrix method," Physical Review E, vol. 75, no. 5, Article ID 056606, 2007. 
[7] J. Yuan, "Computing for second harmonic generation in onedimensional nonlinear photonic crystals," Optics Communications, vol. 282, no. 13, pp. 2628-2633, 2009.

[8] J. W. Haus, R. Viswanathan, M. Scalora, A. G. Kalocsai, J. D. Cole, and J. Theimer, "Enhanced second-harmonic generation in media with a weak periodicity," Physical Review A, vol. 57, no. 3, pp. 2120-2128, 1998.

[9] Y. Dumeige, P. Vidakovic, S. Sauvage et al., "Enhancement of second-harmonic generation in a one-dimensional semiconductor photonic band gap," Applied Physics Letters, vol. 78, no. 20, pp. 3021-3023, 2001.

[10] G. D’Aguanno, M. Centini, M. Scalora et al., "Photonic band edge effects in finite structures and applications to $\mathrm{X}(2)$ interactions," Physical Review E, vol. 64, no. 1, pp. 016609/1016609/9, 2001.

[11] J. Li, Z. Li, Y. Sheng, and D. Zhang, "Giant enhancement of second harmonic generation in poled ferroelectric crystals," Applied Physics Letters, vol. 91, no. 2, Article ID 022903, pp. 1-3, 2007.

[12] Y. Dumeige, I. Sagnes, P. Monnier et al., "Phase-matched frequency doubling at photonic band edges: efficiency scaling as the fifth power of the length," Physical Review Letters, vol. 89, no. 4, pp. 043901/1-043901/4, 2002.

[13] O. V. Shramkova and A. G. Schuchinsky, "Resonant threewave interaction in the nonlinear anisotropic dielectric slabs," in Proceedings of the 5th International Congress on Advanced Electromagnetic Materials in Microwaves and Optics (Metamaterials '11), pp. 757-759, Barcelona, Spain, October 2011.

[14] M. Born and E. Wolf, Principles of Optics, Pergamon Press, Oxford, UK, 1968.

[15] N. Blombergen, Nonlinear Optics: A Lecture Note, Benjamin, New York, NY, USA, 1965.

[16] J. M. Bendickson, J. P. Dowling, and M. Scalora, "Analytic expressions for the electromagnetic mode density infinite, one-dimensional, photonic band-gap structures," Physical Review E, vol. 53, no. 4, pp. 4107-4121, 1996.

[17] Y. Jeong and B. Lee, "Matrix analysis for layered quasi-phasematched media considering multiple reflection and pump wave depletion," IEEE Journal of Quantum Electronics, vol. 35, no. 2, pp. 162-172, 1999.

[18] J.-J. Li, Z. Y. Li, and D. Z. Zhang, "Second harmonic generation in one-dimensional nonlinear photonic crystals solved by the transfer matrix method," Physical Review E, vol. 75, no. 5, Article ID 056606, pp. 1-7, 2007.

[19] P. Szczepanski, T. Osuch, and Z. Jaroszewicz, "Modeling of amplification and light generation in one-dimensional photonic crystal using a multiwavelength transfer matrix approach," Applied Optics, vol. 48, no. 28, pp. 5401-5406, 2009.

[20] J.-J. Li, Z. Y. Li, and D. Z. Zhang, "Nonlinear frequency conversion in two-dimensional nonlinear photonic crystals solved by a plane-wave-based transfer-matrix method," Physical Review B, vol. 77, no. 19, Article ID 195127, pp. 1-5, 2008.

[21] F. G. Bass and A. A. Bulgakov, Kinetic and Electrodynamic Phenomena in Classical and Quantum Semiconductor Superlattices, Nova Science, New York, NY, USA, 1997.

[22] A. Yariv and P. Yeh, Waves in Crystals: Propagation and Control of Laser Radiation, Wiley, New York, NY, USA, 1984. 

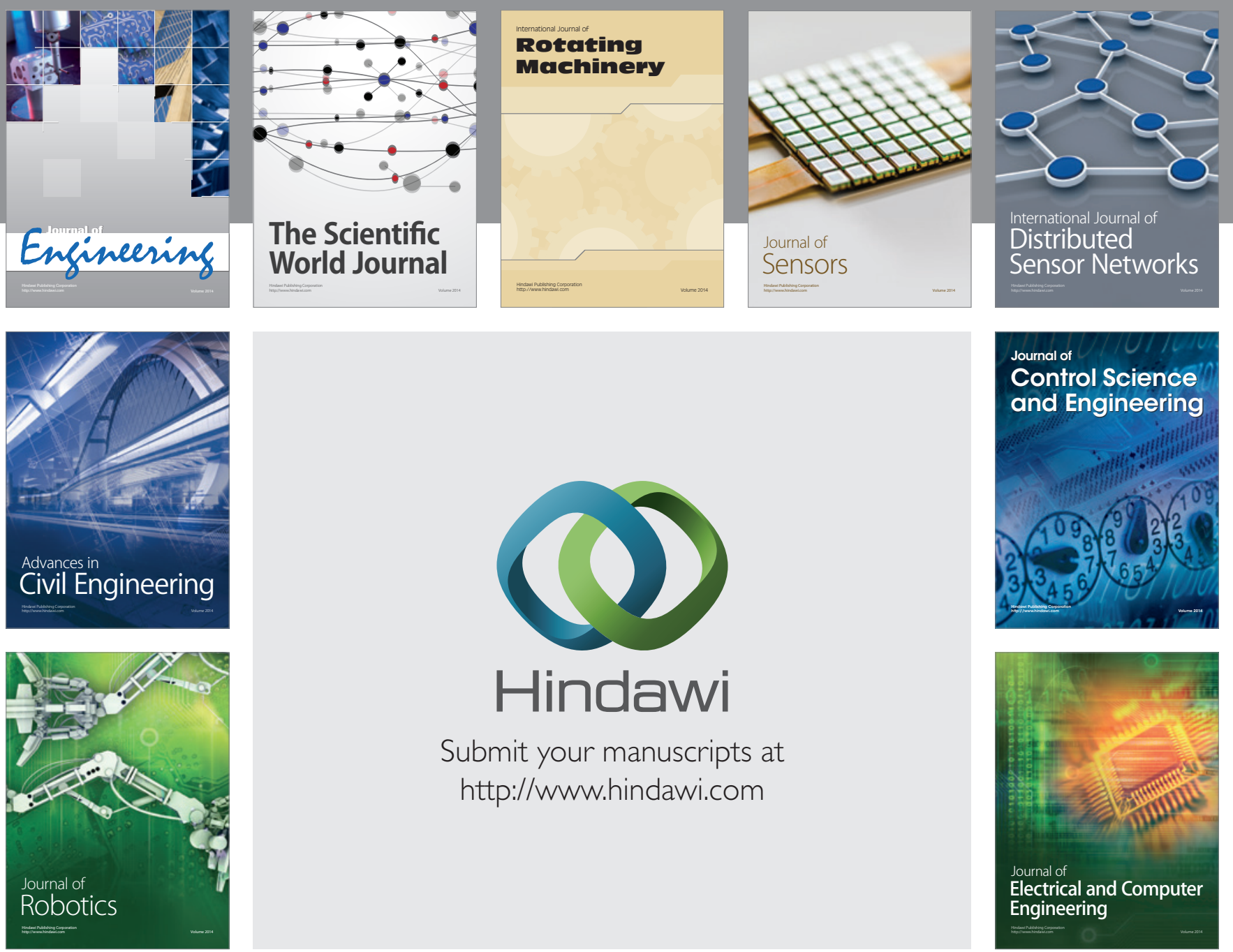

Submit your manuscripts at

http://www.hindawi.com
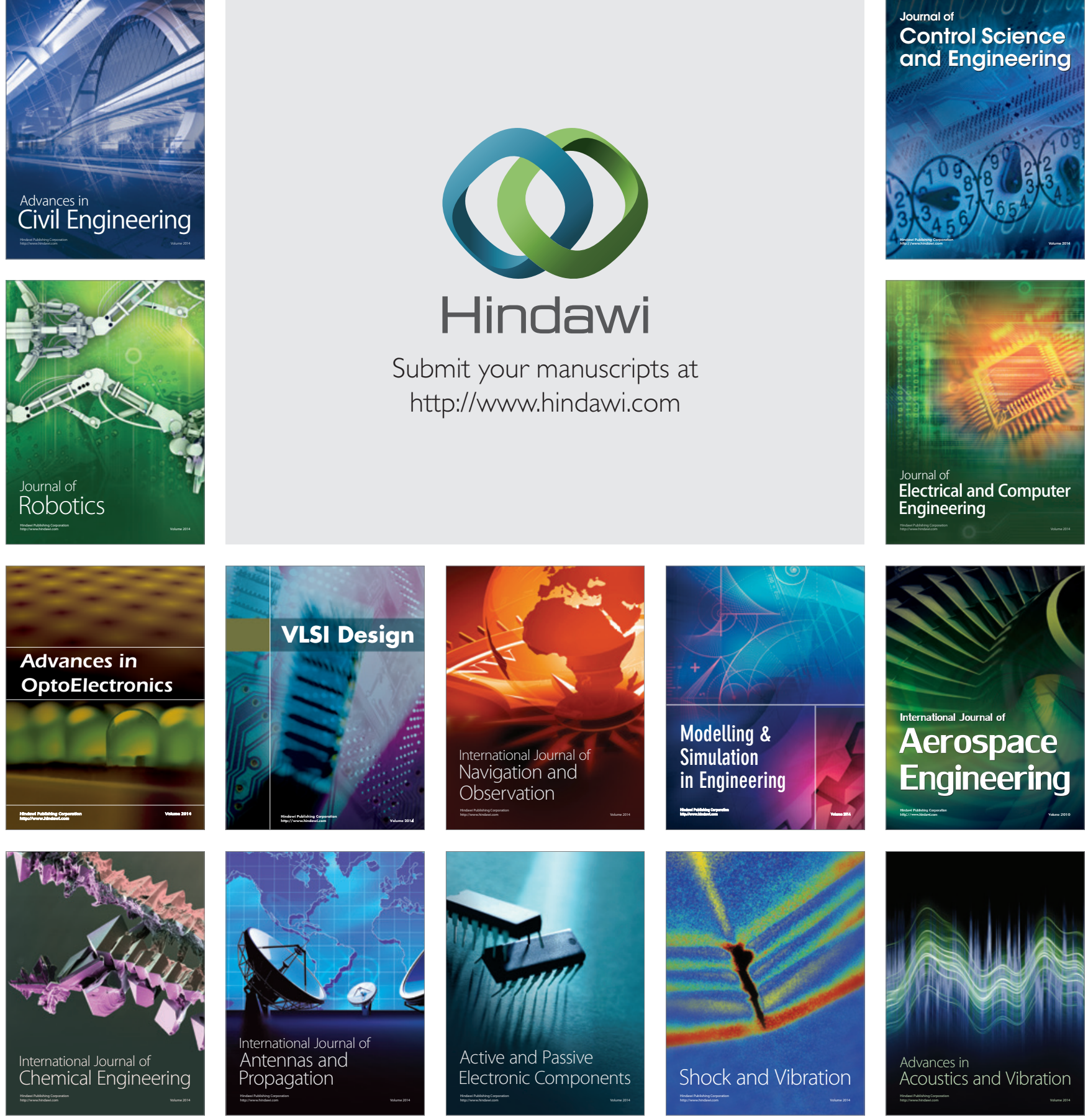